\section{Cahiers de Narratologie}

Analyse et théorie narratives

$19 \mid 2010$

Images composites, arts pluriels

\title{
Optique moderne et post-moderne dans la composition d'images transculturelles dans trois films de Woody Allen
}

Nadia Fuchs

\section{OpenEdition}

Journals

Édition électronique

URL : http://journals.openedition.org/narratologie/6165

DOI : 10.4000/narratologie.6165

ISSN : 1765-307X

Éditeur

LIRCES

Référence électronique

Nadia Fuchs, «Optique moderne et post-moderne dans la composition d'images transculturelles dans trois films de Woody Allen », Cahiers de Narratologie [En ligne], 19 | 2010, mis en ligne le 22 décembre

2010, consulté le 01 mai 2019. URL : http://journals.openedition.org/narratologie/6165 ; DOI :

10.4000/narratologie.6165

Ce document a été généré automatiquement le 1 mai 2019.

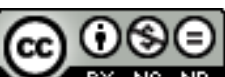

Cahiers de Narratologie - Analyse et théorie narratives est mis à disposition selon les termes de la licence Creative Commons Attribution - Pas d'Utilisation Commerciale - Pas de Modification 4.0 International. 


\title{
Optique moderne et post-moderne dans la composition d'images transculturelles dans trois films de Woody Allen
}

\author{
Nadia Fuchs
}

1 On trouve une des premières références faites par Woody Allen à un autre art que celui du cinéma dans un spectacle de stand-up filmé par la chaîne télévisée britannique BBC en 1965. Son personnage (que le spectateur avait déjà tendance à identifier au cinéaste puisque celui-ci rapportait sur scène des anecdotes biographiques en tant que Woody Allen lui-même) raconte son premier contact direct et charnel avec la peinture. Il se produisit au Metropolitan Museum de New-York:

Un jour, j'ai vu sur un mur un nu de Rubens, mais un nu vraiment succulent ! une chasseresse nue qui poignardait une bécasse. A ce moment-là, j'ai partagé un instant très intense avec ce tableau, et deux gardes ont dû intervenir car je me suis mis à lécher l'huile sur la toile ${ }^{1}$

2 Dans les films de Woody Allen, les artistes sont très présents dans la diégèse, notamment par la présence de personnages tels que ceux de peintres (Frederik dans Hannah and her Sisters, 1986, ou Maria Elena et Juan Antonio dans Vicky-Cristina-Barcelona, 2008), de musiciens (Emmett Ray, guitariste de jazz dans Sweet and Lowdown, 1999), d'écrivains (Harry dans Deconstructing Harry, 1997), ou encore de cinéastes (Sandy Bates dans Stardust Memories), pour n'en citer que quelques-uns.

Mais son cinéma est composite et composé d'autres arts dans la mesure où ils l'imprègnent, surtout sous forme d'intertextualité et de métatextualité, notamment au sens où l'entend Christian Metz :

Avec le redoublement parfait, le retournement sur soi-même, le film « premier » se tisse à travers un autre qui n'est pas vraiment autre, et qui n'est pas vraiment "dans » l'autre. Malheureusement, «film à travers film» est une expression impossible. Pourtant, le rapport des deux couches textuelles est bien de traversée, d'imbrication, de tresse ou d'enchevêtrement, à la limite de la symbiose ${ }^{2}$ 
et de transculturalité, puisque ses films sont des "textes" au sens étymologique du terme, c'est-à-dire qu'ils sont tissés à partir d'autres textes de la littérature, du cinéma, du théâtre, de la danse, des arts picturaux, de la musique, ainsi que des appareils critiques sur ces arts. Néanmoins le choix des fils qui composent ce texte allénien est loin d'être aléatoire, et je vais essayer de démontrer en étudiant trois exemples qu'il utilise surtout d'autres textes modernes, pour en faire une oeuvre finalement post-moderne et transculturelle.

En 1965, Woody Allen finit ainsi son anecdote sur sa découverte de l'art pictural : il décide de trouver un modèle féminin qui posera nue pour lui. Une jeune personne se présente à sa porte:

On sonne à ma porte et je vois une femme fabuleuse, mais vraiment incroyable ! Je l'invite tout de suite à entrer, et je ferme la porte à double tour. [...] Et je lui dis : "Déshabillez-vous ", car je ne connais pas grand chose à l'art, mais je sais ce que j'aime. Elle enlève tout, très professionnelle et se met à poser. Et là je me mets à trembler. [...] Je transpire bruyamment. Elle se tient devant moi, majestueuse, je m'avance vers elle avec ma feuille de papier et mon fusain, et c'est là que j'ai rencontré un petit problème avec elle parce que j'ai essayé de la décalquer³.

Ce dernier verbe, "to trace" en anglais signifie à la fois "décalquer" et "faire le contour ", et ces deux sens illustrent très bien deux stratégies énonciatives, filmiques et cinématographiques utilisées dans la majorité des films du cinéaste : d'une part celle du cadrage et de l'encadrement pour mettre en lumière le dispositif, pour briser l'illusion spectatorielle et créer un effet de distanciation (tel que l'ont utilisé les modernistes comme Bertolt Brecht et son Verfremdungseffekt notamment, et le critique littéraire formaliste russe Viktor Chklovski qui préconise la « défamiliarisation » d'un objet ou d'un personnage, visant à créer un effet d'étrangeté pour que le lecteur ou le spectateur ne puisse pas s'identifier complètement au personnage et puisse prendre conscience de ce qu'il lit ou voit).

7 Le second procédé très utilisé dans l'œuvre par Woody Allen, évoquée par ce décalquage, renvoie à l'idée de copie, de copie de copies et de la notion d'hyperréalité développée par Jean Baudrillard dans son ouvrage Simulacres et simulation. ${ }^{4}$. En parlant du rôle du signe dans le monde occidental, il affirme que

Toute la foi et la bonne foi occidentale se sont engagées dans ce pari de la représentation : qu'un signe puisse renvoyer à la profondeur du sens, qu'un signe puisse s'échanger contre du sens et que quelque chose serve de caution à cet échange - Dieu bien sûr. Mais si Dieu lui-même peut-être simulé, c'est-à-dire se réduire aux signes qui en font foi ? Alors tout le système passe en apesanteur, il n'est plus lui-même qu'un gigantesque simulacre - non pas irréel, mais simulacre, c'est-à-dire ne s'échangeant plus jamais contre du réel, mais s'échangeant en luimême, dans un circuit ininterrompu dont ni la référence ni la circonférence ne sont nulle part ${ }^{5}$.

Il définit ensuite trois niveaux de simulations :

- le premier est une copie de la réalité.

- Le deuxième est une copie tellement bonne qu'on ne voit plus la frontière entre la réalité et la représentation.

- Le troisième niveau produit une réalité par elle-même, où il n'y a presque plus de référence au monde réel.

- Il s'agit donc d'une copie d'une copie, dans laquelle on ne garde que le signe, le contour, et dans une perspective transculturelle, le cliché. 
Lors d'un entretien accordé à Times/CNN en janvier 2008 intitulé «Ten Questions for Woody Allen ", un téléspectateur posait au cinéaste la question suivante: "Etes-vous d'accord avec Picasso qui disait que les bons artistes copient, les grands volent?». Sa réponse fut la suivante : «Oh j'ai volé aux meilleurs [on notera au passage qu'il se classe directement dans la catégorie des grands]. J'ai volé à Bergman. J'ai volé à Groucho, Chaplin, Keaton, Martha Graham, Fellini. Je suis un voleur sans aucun scrupule ${ }^{6}$ »

Notre premier exemple est justement une référence directe au film de Fellini Otto e Mezzo (Huit et demi, 1963). On se souvient de la séquence d'ouverture composée de plans fixes sur des automobilistes coincés dans un embouteillage. Les personnages sont filmés en plans serrés, de face, depuis l'extérieur, et portent pour la plupart des lunettes. De sorte qu'ils sont triplement encadrés : par le cadre de l'image, par celui du pare-brise, et enfin par celui de la monture de leurs lunettes, larges et noires, à la mode dans les années soixante.

11 Le film Stardust Memories (1980) fait un emprunt direct à Fellini. La première séquence montre en fait le nouveau film réalisé par Sandy Bates (lui-même joué par Woody Allen) dans lequel il joue le rôle principal, et qui est projeté pendant un festival qui lui est consacré.

12 Les personnages ne sont pas dans un embouteillage mais dans un train ou plutôt dans deux trains. Dans celui où se trouve le personnage joué par Sandy Bates, l'atmosphère est assez sombre, les acteurs sont filmés de face, et portent aussi souvent le même type de lunettes. Soudain, l'acteur Sandy Bates regarde par la fenêtre et voit un autre train allant dans la même direction dans lequel les gens boivent du champagne et font une fête. Une jeune femme lui fait un signe par la fenêtre. Un premier effet de distanciation est créé par le fait que le regard des acteurs s'adresse directement au spectateur. De plus, ils sont euxmêmes cadrés par les fenêtres des wagons, qui avancent, et finalement, cette succession de cadres de fenêtres qui défilent sous les yeux de Sandy Bates/ Woody Allen n'est pas sans évoquer une pellicule de film et ses photogrammes.

13 Nous avons donc ici une mise-en-abyme d'une mise-en-abyme en quelque sorte puisque Stardust Memories, en évoquant justement le souvenir cinématographique du film italien, reprend son procédé de distanciation et de défamiliarisation.

14 Les deux exemples suivants sont deux films, qui par leur titre sont ostensiblement des adaptations d'œuvres littéraires. Le premier est le film de 1975 Love and Death (Guerre et Amour) qui fait évidemment référence au roman de Tolstoï Guerre et Paix. Il s'agit d'une comédie, dans laquelle Woody Allen et Diane Keaton jouent les rôles principaux de Boris et Natacha., et la trame diégétique suit celle du roman russe. Mais ce qui est singulier dans cette adaptation est qu'elle emprunte des éléments et notamment des images aux formalistes russes des années 20 , et au cinéma soviétique en général.

La bande-son est en fait la musique composée par Prokofiev pour le film d'Eisenstein Alexandre Nevski (1938), ce qui est donc un détournement de son objet initial.

Par ailleurs, on trouve une référence visuelle directe au Cuirassé Potemkine (1925) du même Eisenstein bien sûr. Après la célèbre séquence du massacre du peuple sur le grand escalier d'Odessa, le cuirassé tire des coups de canon sur le quartier général des militaires, et pour symboliser ce soulèvement, trois plans de la statue d'un lion se succèdent. La sculpture de ce lion est posée sur une colonne. Le premier plan le montre affalé sur la colonne, les pattes pendantes, dans le deuxième il est assis, et à la fin il est dressé sur ses pattes avant. 


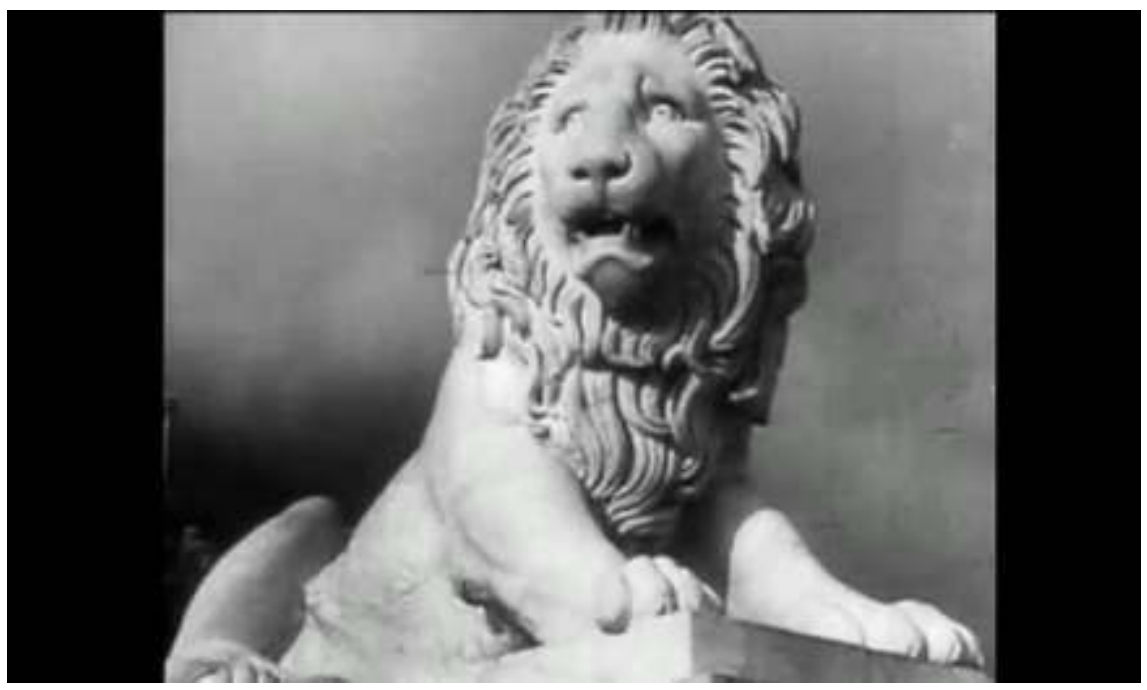

Le Cuirassé Potemkine, B. Eisenstein [dr]

17 Cet insert de trois plans montre bien l'utilisation par Eisenstein du Verfremdungseffekt de Brecht, puisque le récit est interrompu par cette animation d'une statue qui devient métaphore de soulèvement du peuple, et même temps montre au spectateur l'énonciation militante du cinéaste.

Love and Death emprunte ces trois plans à des fins différentes. Ils sont utilisés comme insert dans la séquence où Boris et Natacha consument leur relation dans un lit. Boris enfile des gants de boxe, et en lieu et place d'une représentation de relation sexuelle, on voit les trois mêmes plans avec une statue de lion très similaire, mais dans l'ordre inverse. Le lion dressé et victorieux au début est écroulé et épuisé sur la colonne à la fin.

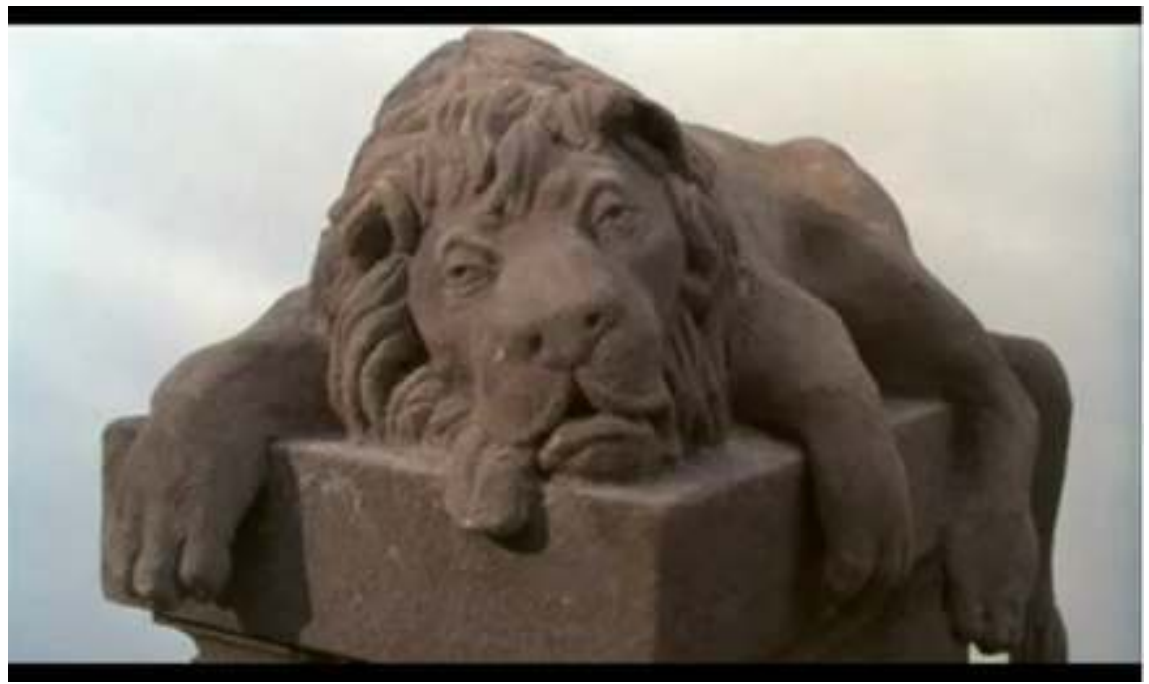

Love and Death, Woody Allen [dr]

19 La composition de cette séquence a trois fonctions :

- une fonction évidemment comique.

- une fonction d'hommage aux films d'Eisenstein

- et enfin un parti-pris dans l'adaptation cinématographique du roman de Tolstoï, puisque Woody Allen choisit délibéremment des artistes modernes non seulement de la même sphère culturelle russe, mais aussi soviétique, à l'avant-garde de leur époque. 

ennuyeuse, peuplée de boutiques de luxe, de salons de coiffure haut de gamme et de conversations avec d'autres femmes de ce milieu sur la dernière boutique à la mode ou la nouvelle opération de chirurgie esthétique subie par l'une d'elles. Alice développe un mal de dos psychosomatique et se rend chez un médecin acuponcteur, le docteur Yang, dont le cabinet se trouve à Chinatown. Les traitements qu'il va lui prescrire ressemblent fort à ceux de l'Alice de Lewis Carroll, puisque ce sont des potions et autres décoctions qui vont la transformer physiquement, et lui permettre de savoir qui elle est ou du moins ce qu'elle considère comme important dans la vie. Elle finira par divorcer et aller travailler avec Mère Térésa à Calcutta, avant de revenir s'installer dans un quartier populaire de New-York.

trouve aussi des personnages de Lewis Carroll, comme le Chat du Cheshire, ici représenté sous la forme du fantôme de son premier amour décédé, qui apparaît et disparaît progressivement tout en faisant entendre son rire. Le Docteur Yang rappelle aussi le Ver à soie qui lui pose des questions existentielles, fume la cigarette puis le narguilé, et joue énormément sur la signification du langage.

Mais Allen, dans son adaptation de l'œuvre de Carroll, reprend aussi ses procédés, notamment différents discours de son époque, qui en font un texte dialogique, au sens où l'entend Mikhail Bakhtine'. En effet, Carroll met dans la bouche de ses personnages du monde des merveilles une multitude de discours de son époque, qu'il parodie et pastiche, notamment des discours politiques, des comptines enfantines, des procès à la cour, parmi d'autres.

Woody Allen- va reprendre des discours et des langages cinématographiques, et ceci, dès la première séquence du film. Alice se trouve avec un homme brun (qui n'est pas son mari) au zoo devant des aquariums contenant des pingouins, et l'embrasse. La séquence deux montrera qu'en fait, il s'agissait d'une séquence de rêve, de somnolence, comme l'Alice de Carroll qui s'assoupit au bord de l'eau. Cette séquence de pingouins peut faire référence aux premiers moments d'Alice qui, en arrivant au pays des merveilles, pleure tellement que ses larmes forment un lac dans lequel se baigne une multitude d'animaux. Mais c'est aussi un emprunt au film d'Orson Welles The Lady from Shanghai (1947). Michael o'Hara (Orson Welles) retrouve sa maîtresse Elsa Bannister (Rita Hayworth) dans un zoo, précisément devant des aquariums, non pas peuplés de pingouins mais de poulpes et autres poissons bien plus menaçants et annonciateurs du complot contre Arthur Bannister. Les séquences sont très similaires filmiquement, et les couples qui s'embrassent sont encadrés et enfermés par la vitre des aquariums. 


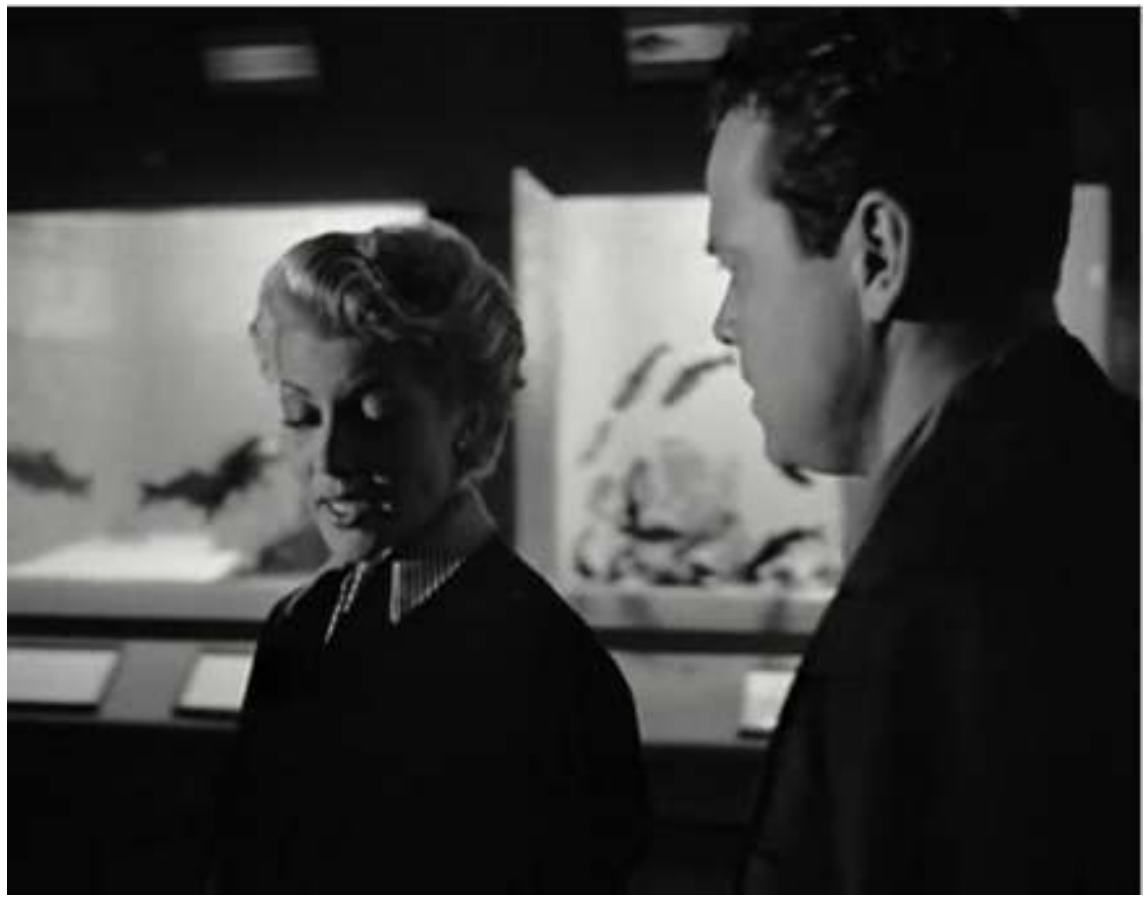

The Lady from Shanghaï, Orson Welles [dr]

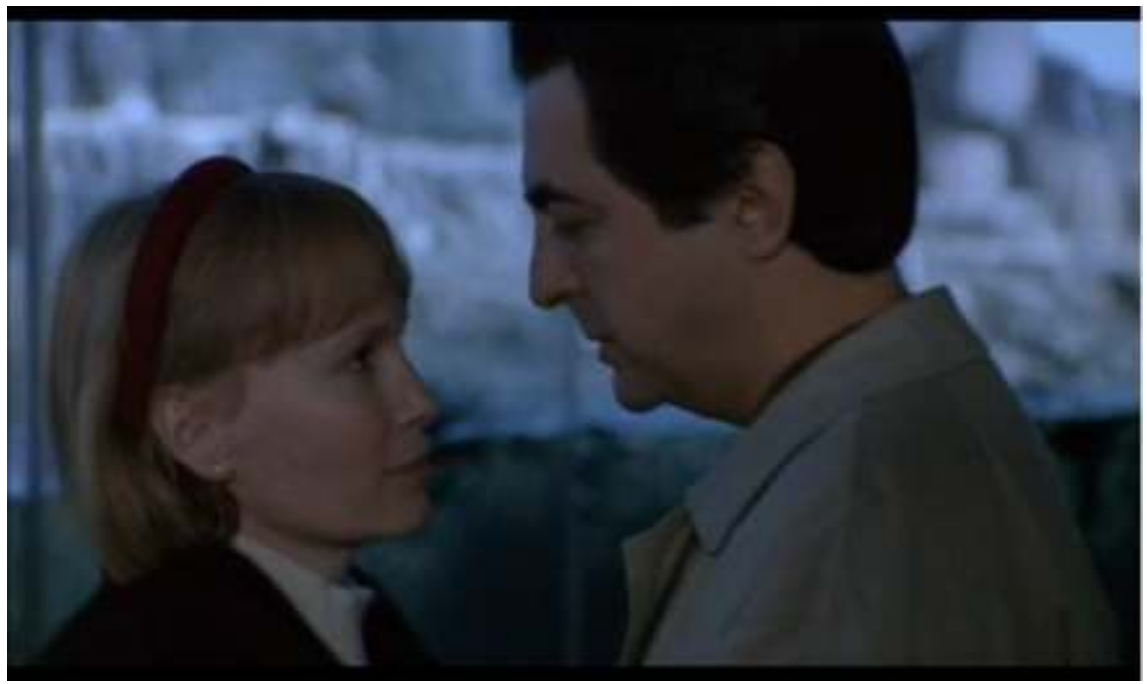

Alice, W. Allen $[\mathrm{dr}]$

Cette référence intertextuelle est d'autant plus intéressante, que l'équivalent du Wonderland de Carroll va précisément être le Docteur Yang et Chinatown, ce monde très exotique pour Alice. C'est un cliché orientaliste qui revient dans beaucoup de films de Woody Allen comme représentant l'Autre, et qui est présent aussi dans le roman de Carroll. C'est d'ailleurs lorsqu'Alice se rend chez le Docteur Yang que symboliquement, elle va tomber dans le terrier du lapin. En effet, le docteur chinois l'hypnotise en utilisant une roue à spirale (" a vortex » en anglais) qui crée une illusion d'optique de profondeur et donc de chute. 


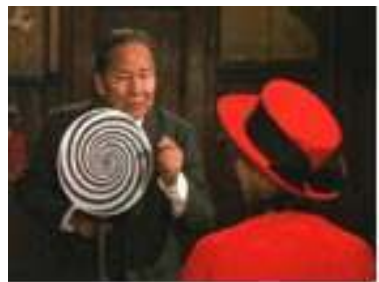

Alice, W. Allen [dr]

27 Ici, le discours "emprunté » est celui d'une autre adaptation d'Alice, celle de Disney (1951) dans laquelle le passage d'Alice du monde réel à celui des merveilles est représenté par des cercles concentriques qu'Alice fait dans la rivière dans lesquels se reflètent soudain le lapin blanc.

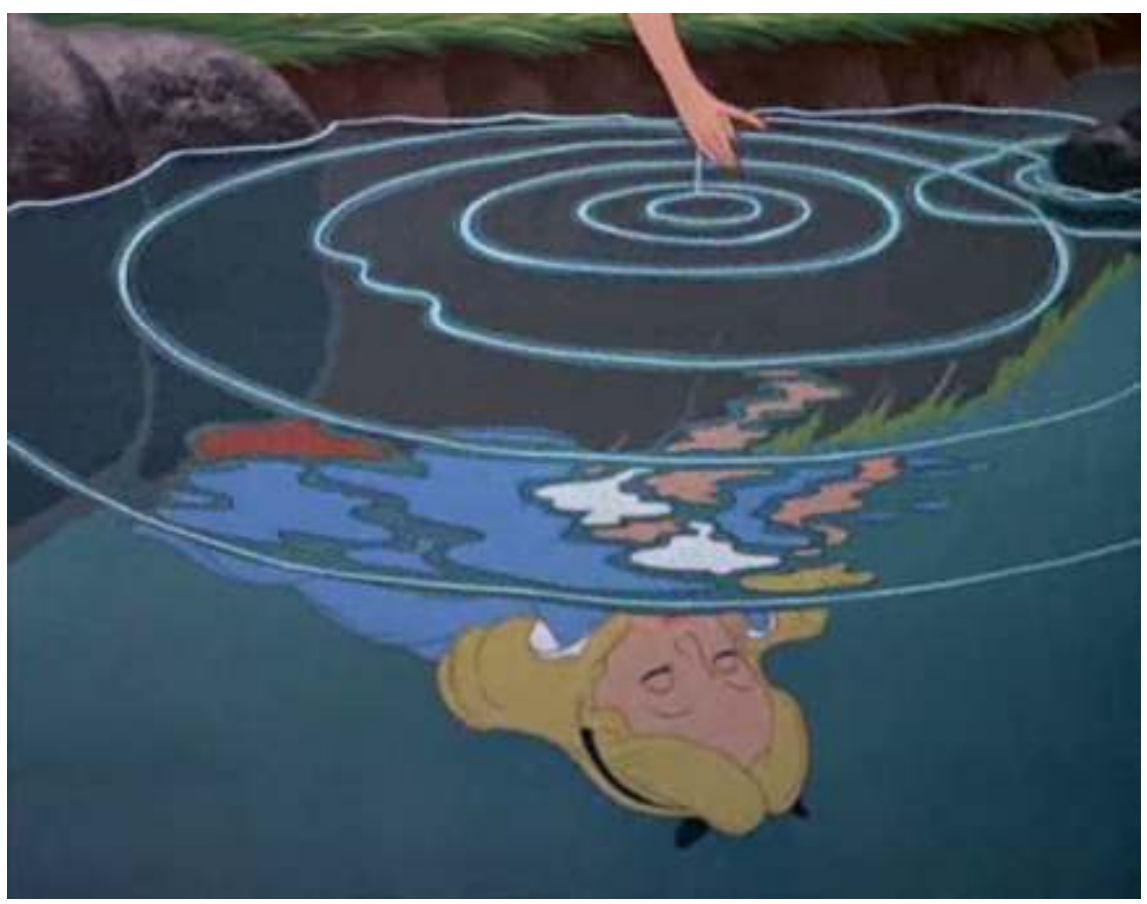

Alice, C. Geronimi, W. Jackson, -H. Luske, Studios Disney [dr]

Ici, donc, le film mélange culture savante et culture populaire dans une démarche complètement post-moderne.

Un autre exemple de ceci est visible lorsque l'Alice allénienne passe du temps avec son nouvel amant, et se promène au sommet d'un building à New-York qui fait face à de gigantesques panneaux publicitaires, notamment pour la marque de cigarettes Camel. 


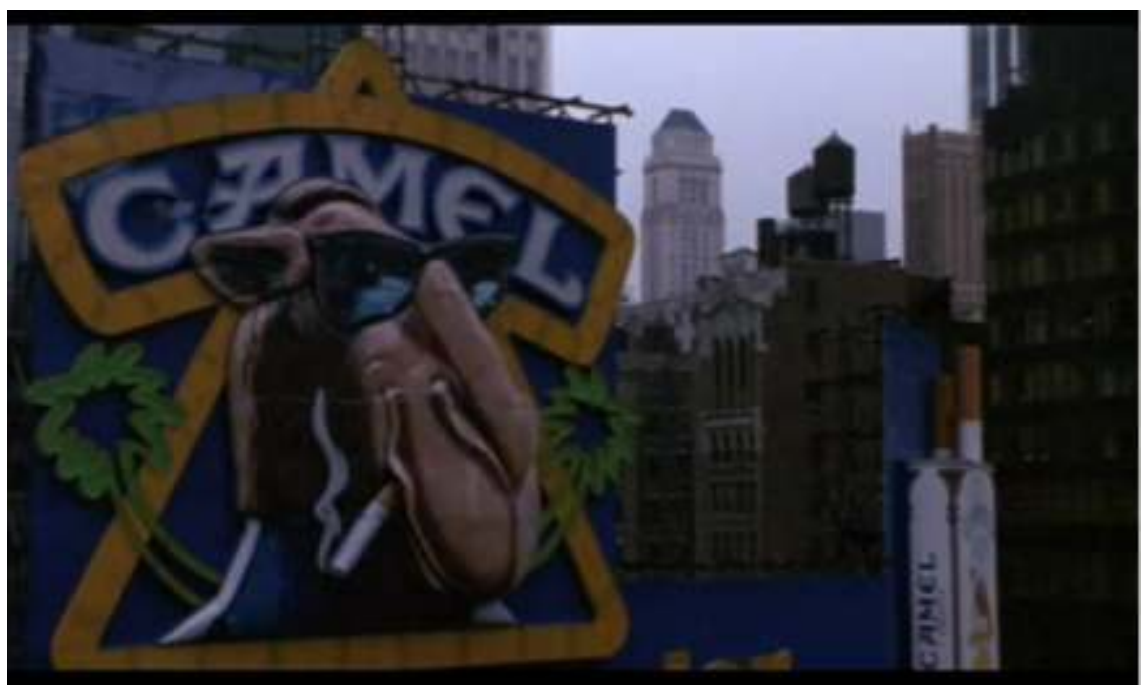

Alice, W. Allen $[\mathrm{dr}]$.

30 Dans ce plan, nous pouvons voir plusieurs évocations. D'une part, une équivalence d'une créature étrange du Wonderland (wonder qui ne signifie pas exactement " merveilles » en anglais, mais plutôt des choses « surprenantes»), et on pourrait entendre la petite fille se poser bien des questions sur ce chameau sur un mur portant des lunettes de soleil et fumant une cigarette. D'autre part, ce panneau illustre l'idée de Baudrillard de la copie qui a perdu sa référence au réel, car qui pense aujourd'hui au fait que le chameau est là pour montrer que le papier de ces cigarettes provenait à l'origine de Turquie, et donc évoquait déjà un orientalisme moins lointain?

31 La dernière image composite sur laquelle nous allons nous attarder est l'image de la muse qui apparaît devant Alice dans le film de Woody Allen, lorsqu'elle décide de se mettre à écrire. Le Docteur Yang lui a donné une tisane, et après l'avoir bue, un nouveau personnage se présente à elle.



Alice, W. Allen [dr]

32 Cette muse apparaît sous un spot lumineux, et affirme à Alice qu'elle est sa muse. Son apparence fait tout de suite penser à la Flore du Printemps de Botticelli (1492), habillée de la même façon, et cette référence est confirmée par le fait que cette muse parle avec un accent italo-américain extrêmement prononcé. De plus, on retrouve dans l'Alice de 
Disney un personnage semblable sous la forme de la star des roses qui chantent dans le jardin enchanté.

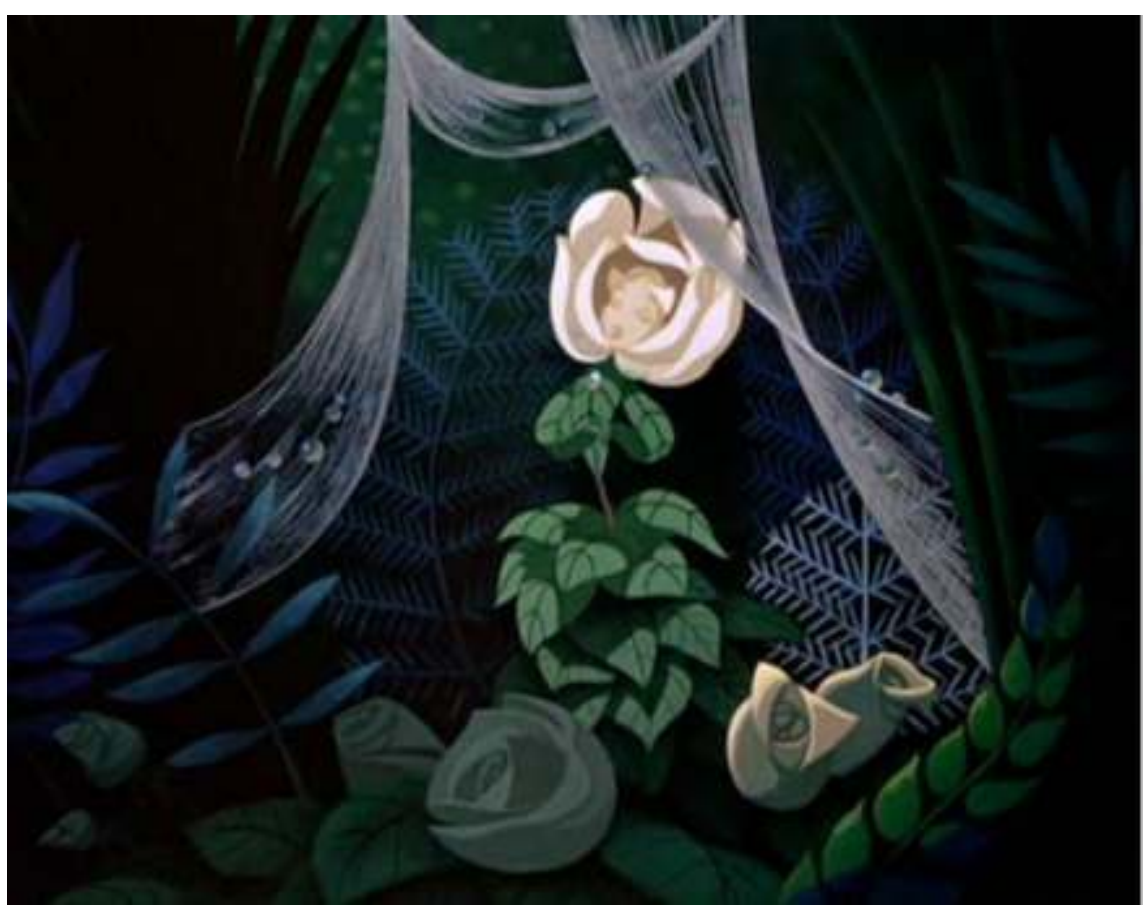

Alice, C. Geronimi, W. Jackson, -H. Luske, Studios Disney [dr].

Nous voyons ici donc à la fois ce phénomène de distanciation et de défamiliarisation, non seulement par le cadre mais aussi par les clichés culturels (l'Italianité, comme aurait dit Roland Barthes), et le mélange post-moderne des genres et des références.

Le cadre ultime apparaît lorsque cette muse, pour lire le manuscrit d'Alice, revêt des lunettes à larges montures noires, symboles cette fois-ci de son " allenité » (cet accessoire est en effet devenu au fil des films le signe de la présence du personnage « Woody » et du jeu qu'il joue avec le spectateur sur son propre statut de personnage/célébrité/personne réelle).

Woody Allen, dans ces films-ci comme dans d'autres, choisit ses références non seulement dans le cinéma mondial, mais aussi dans les autres arts modernes, et déconstruit ce que nous prenons pour le réel à la manière de Roland Barthes dans Mythologies.

Comme Norman K. Denzin, dans son ouvrage Images of Postmodern Society ${ }^{8}$, on peut considérer que Woody Allen est un post-moderne conservateur, dans la mesure où son hyperréalisme est tissé de références à un passé historique :

Le projet d'Allen consiste à raconter et à généraliser ses histoires bizarres, personnelles, ethniques, familiales, sexuelles, fantasmatiques, et à les transformer en une série de textes de culture populaire qui propose une interprétation radicale, humoristique de la condition post-moderne et la déconstruit. [...] Elle célèbre le voyeur en tant que figure culturelle qui, par son regard insistant, devient touriste dans l'espace contemporain. Tel un charognard, ce voyeur est à la recherche d'éléments significatifs dans les déchets culturels du passé et du présent qui encombrent la vie quotidienne. [...] Comme Erasme, ses films font l'éloge de la folie humaine, tout en prenant très au sérieux ce moment post-moderne ${ }^{9}$ 


\section{NOTES}

1. And I saw on the wall, once, a nude by Rubens, but a real succulent nude, a naked huntress stabbing to death a woodcock. And I got very emotionally involved with the painting. Two guards had to restrain me. I tried to lick some of the oil off the canvas.

2. Christian Metz, L'énonciation impersonnelle ou le site du film, Paris : Méridiens Klincksieck, 1991.

3. There's a knock on my door and standing there is this fabulous woman, but really sensational ! I let her in quickly, and I lock the door. [...] And I said to her "Take off your clothes", because I don't know much about art but I know what I like. She took everything, very professional and posed. And I began to shake. I'm perspiring audibly. She's standing there in front of me, majestic, and I took my piece of paper and my charcoal pencil, and I went up to her, and I got into a little troulbe with her because I tried to trace her."

4. Jean Baudrillard, Simulacres et simulation, Paris : Gallilée, 1981.

5. Ibid. page 16.

6. Times/CNN, 17 janvier 2008.

«- Do you agree with Picasso's quote : "Good artists copy ; great artists steal" ? -Debbie Johnson, Rochester, Mich.

-Oh, I've stolen from the best. I've stolen from Bergman. I've stolen from Groucho, from Chaplin, from Keaton, from Martha Graham, from Fellini. I mean I'm a shameless thief. »

7. Mikhail Bakhtine, La poétique de Dostoïevski, Paris : Seuil, 1970.

8. Norman K . Denzin, Images of Postmodern Sociey, London : Sage, 1991.

9. Ibid. page 105: «Allen's project consists of the telling and generalization of his peculiar, personal, ethnic, familial, sexual, fantasy stories into a series of popular culture texts which offer a radical, humorous, deconstructive interpretation of the postmodern condition. [...] It celebrates the voyeur as a cultural figure who, in his (and her) looking, becomes a tourist of the contemporary scene. Like a scavenger, this voyeur searches for meaningful items in the cultural refuse of the past and the present that litters everyday life. [...] Like Erasmus, his works praise human folly, while they take dead seriously this postmodern moment. »

\section{RÉSUMÉS}

Avec le personnage récurrent juif new-yorkais névrosé plus ou moins autobiographique, les films de Woody Allen ont créé, volontairement ou pas, leur propre mythologie et un genre à part entière composé de codes que le spectateur décrypte instantanément. Pourtant, Woody Allen est surtout un cinéaste post-moderne, analysant et déconstruisant les simulacres de son époque. Il est ainsi un passeur d'images retraçant l'histoire des arts modernes des XXe et XXIe siècles et de leurs stratégies énonciatives et réceptives.

With the recurrent persona of the neurotic and more or less autobiographical Jewish New Yorker, Woody Allen's films have created, willingly or not, their own mythology and a specific 
genre made up of codes readily accessible to the spectator. However, Woody Allen is more than anything else a postmodern filmmaker who analyses and deconstructs the simulacra of his time. He is thus a conveyor of images which retrace the history of modern art of the XXth and XXIth centuries, and of their strategies of enunciation and reception.

INDEX

Mots-clés : distanciation, Eisenstein, hyperréalité, intertextualité, Lewis Carroll, modernisme, post-modernisme, transculturalité, Woody Allen

\section{AUTEUR}

NADIA FUCHS

Université Nice-Sophia Antipolis 